\title{
Bullying homofóbico y vulnerabilidad de estudiantes LGBT+ en escuelas brasileñas: un ensayo crítico*
}

\author{
Emerson Vicente da Cruz ${ }^{* *}$ \\ Henrique Caetano Nardi*** \\ Elka Lima Hostensky**** \\ Lucas Rech da Silva***** \\ Esteve Espelt ${ }^{* * * * *}$
}

\section{Resumen}

La experiencia escolar puede desempeñar un papel en el aprendizaje de conceptos que promueven la igualdad y la deconstrucción de los mitos que constituyen un referente normativo en el que se articulan los discursos y prácticas que sostienen las opresiones. Este ensayo, que trata el tema de la violación de los derechos humanos LGBT+ y el impacto de acciones institucionales sobre la salud y el bienestar LGBT+ en el ámbito escolar brasileño, se divide en dos partes: en la primera, se presentarán las políticas educativas contra la LGBTfobia desde la introducción del programa Brasil

\footnotetext{
* El primer autor del presente estudio es miembro del Grupo de Investigación en Psicologia Social y Organizacional PsicoSAO (2017SGR564) de la Universitat de Barcelona. Este articulo se publica con el soporte de PsicoSAO.

** Departament de Psicologia Social i Psicologia Quantitativa, Universitat de Barcelona.

*** Universidade Federal do Rio Grande do Sul (UFRGS), Instituto de Psicologia.

**** Universidade Estadual de Santa Catarina (UFSC) Florianópolis (SC)

***** Pontifícia Universidade Católica do Rio Grande do Sul (PUCRS), Programa de Pósgraduação em Educação.

****** Departament de Psicologia Social i Psicologia Quantitativa, Universitat de Barcelona (UB).
} 
sem Homofobia. Luego, se analizará el bullying homofóbico como fruto de la vulneración de los derechos LGBT+. Este trabajo advierte que la privación de los derechos LGBT+ afecta a la construcción de su identidad e imagen psicosocial y destaca el valor de coeducar en espacios amables, desde el respeto y la aceptación a la diversidad.

Palabras clave: Bullying homofóbico. Derechos humanos LGBT+. Educación. Diversidad afectivo-sexual y de género. Lenguaje homofóbico.

\section{Homophobic bullying and vulnerability of LGBT+ students in Brazilian schools: a critical essay}

\section{Abstract}

School can offer pupils a chance to acquire concepts that promote equality, as well as deconstructing the myths that underpin a normative framework on which the discourses and practices that sustain oppression are built. This essay examines the violation of LGBT+ human rights and the impact that government-led initiatives can have on pupils' health and well-being in Brazilian context, and is structured in two parts: firstly, educational policies targeted against LGBTphobia will be analyzed drawing on the implementation of the Brasil sem Homofobia program. Subsequently, homophobic bullying will be analyzed as an outcome of the violation of LGBT+ rights. This study draws attention to the fact that depriving the LGBT+ population of their rights damages the construction of their identity and psychosocial image, highlighting the value of coeducation in safe spaces, nurturing respect and acceptance of diversity.

Keywords: Affective-sexual and gender diversity. Education. Homophobic bullying. Homophobic language. LGBT+ human rights.

\section{Bullying homofóbico e vulnerabilidade de estudantes LGBT+ em escolas brasileiras: um ensaio crítico}

\section{Resumo}

A experiência escolar pode desempenhar um papel na promoção de conceitos que promovam a igualdade, e a desconstrução dos mitos que constituem um referencial normativo em que se articulam os discursos e as práticas que sustentam as opressões. Este ensaio, que trata o tema da violação dos direitos humanos LGBT+ e o impacto que ações institucionais têm sobre a saúde e o bem-estar LGBT+ no âmbito escolar brasileiro, 
estrutura-se em duas sessões: na primeira, serão apresentadas as políticas educativas voltadas ao combate à LGBTfobia desde a implementação do programa Brasil sem Homofobia. Logo, se analisará o bullying homofóbico como fruto da vulneração dos direitos LGBT+. Este trabalho alerta que a privação dos direitos da população LGBT+ afeta na construção da sua identidade e imagem psicossocial e ressalta o valor de coeducar em espaços amáveis, desde o respeito e aceitação à diversidade.

Palavras chaves: Bullying homofóbico. Direitos humanos LGBT+. Educação. Diversidade afetivo-sexual e de gênero. Linguagem homofóbico.

\section{Introducción}

Brasil llegó a la $7^{a}$ posición entre las potencias económicas del mundo en las primeras décadas del siglo XXI y, acompañando ese crecimiento económico, se implementaron varias políticas públicas para fortalecer los derechos humanos. Hoy en día, Brasil está viviendo tiempos difíciles tanto en el plano económico como en lo referente a retrocesos en los derechos sociales. Además, el pasado colonial y esclavista, marcado por la fuerte presencia de religiones con tradiciones judeocristianas, dejó cicatrices históricas con respecto a una jerarquía del ser humano, afectando el acceso a los derechos sociales en una sociedad altamente desigual. Así pues, aunque la Constitución de 1988, llamada la "Ciudadana", sentó las bases para la construcción de una sociedad más justa, los derechos sociales, condición esencial de los derechos humanos ${ }^{1}$, a menudo son amenazados, especialmente en lo referente a grupos sociales "minoritarios" (NARDI; QUARTIERO; RODRIGUES, 2020). Entre estos grupos encontramos la población de Lesbianas, Gais, Bisexuales, Transexuales y Travestis, junto con todas las demás denominaciones referentes a la diversidad afectivo-sexual y de género (LGBT+).

Aunque la población LGBT+ se representa como una minoría, cuantificar este colectivo es un reto difícil para el Instituto Brasileño de Geografía y Estadística. Se sabe que es “uma população plural

Derechos sociales son aquellos asociados a la ciudadanía (salud, educación, seguridad, vivienda, trabajo, entre otros) y son la base para que los derechos humanos - es decir, aquellos que promuevan la igualdad sin distinción de raza, etnia, edad, sexualidad, género, religión, origen, clase social - sean respetados. 
e diversa, formada por diferentes identidades de gênero e orientações sexuais não normativas que pautam o enfrentamento à violência e outros temas relacionados a direitos a partir das especificidades que cada grupo carrega2" (BULGARELLI et al., 2019, p. 16).

De hecho, mientras no exista igualdad de derechos y la pertenencia al colectivo LGBT+ represente un obstáculo o un riesgo de muerte en Brasil, no será posible cuantificar el colectivo LGBT+ brasileño. Lo que sí se podrá hacer es asegurar que este colectivo social es una minoría en el sentido político, al no ocupar una posición política equivalente a la de la población heterosexual y cisgénera. Hay que destacar que esta población ha construido formas de resistencia cultural, además de ser un grupo consumidor que se encuentra en el punto de mira del mercado llamado pink (rosa), que usa la publicidad y el marketing para "incluir" la diversidad, pero que se resiste a crear puestos de trabajo para esa misma diversidad. Así pues, su representatividad es escasa y eso afecta la forma en que se trata la diversidad en todos los ámbitos de socialización, y sobre todo en el educativo.

Históricamente, la sociedad brasileña ha identificado la población LGBT + a partir de los discursos morales, ideológicos y religiosos, una herencia colonial que se perpetúa a través de la colonialidad del conocimiento, el ser y el poder (NARDI; GONCALVES, 2019). Por este motivo, la experiencia escolar puede desempeñar un papel fundamental en la construcción de aprendizajes que promuevan la igualdad y el respeto a la diversidad, así como la deconstrucción y transformación de mitos sociales que constituyen un referente normativo en el que se articulan los discursos y prácticas que sostienen la posición dominante de diferentes colectivos sociales.

Este ensayo desarrolla teórica y analíticamente un diálogo entre las demandas de la población LGBT+ en Brasil y los estudios so-

2 "una población plural y diversa, formada por diferentes identidades de género y orientaciones sexuales no normativas que orientan la lucha contra la violencia y otras cuestiones relacionadas con los derechos en base a las especificidades propias de cada grupo" 
bre el bullying homofóbico. El objetivo es tratar la vulneración de los derechos humanos LGBT+ y el impacto que acciones institucionales pueden tener sobre la salud y bienestar LGBT + en el ámbito educativo. Para subrayar el enfoque interdisciplinar del presente ensayo crítico, se realizará un diálogo entre la psicología social y las ciencias de la educación, para enriquecer, expandir y problematizar los retrocesos de los derechos humanos LGBT+ en Brasil relacionándolos con el bullying homofóbico desde un abordaje psicosocial con perspectiva de género (VICENTE DA CRUZ et al., 2016).

Este ensayo se estructura en dos partes: primero se presentarán algunas políticas públicas encaminadas a la lucha contra la LGBTfobia desde la implementación del Programa Brasil sem Homofobia, y se presentará el panorama político actual y su relación con la población LGBT+. En la segunda parte se analizará el fenómeno del bullying homofóbico en tanto que resultado de la vulneración de los derechos fundamentales de la población LGBT+.

\section{Lucha contra la homofobia en el contexto edu- cativo brasileño}

La consolidación de los ideales de pluralidad y la equidad de derechos de los grupos minoritarios con poca representatividad política y social (indígenas, quilombeas, LGBT+, etc.) deberían estar entre las prioridades de la actual agenda educativa brasileña dadas las alarmantes tasas de violencia escolar contra estos colectivos, en especial la población LGBT+. La escuela es el espacio en el que estas personas experimentan situaciones de violencia que afectan su salud emocional, su bienestar, su identidad, la forma en que se relacionan con el otro y cómo viven la experiencia escolar. En este sentido, debería haber un compromiso por parte del Estado para promover la salud, el bienestar de la población LGBT+, combatiendo la violencia escolar (BRASIL, 2004), ya que la Constitución Federal de 1988 y la Ley de Directrices y Bases de Educación (LDB, no 9.349/96) prevén el derecho a una escolaridad libre de discriminación.

En 2004, el gobierno federal lanzó el Programa Brasil sem Homofobia (BRASIL, 2004), que ha constituido un importante marco 
político y social y un instrumento para combatir la LGBTfobia durante al menos una década. Su implementación motivó y desencadenó una serie de medidas que tuvieron impacto en el ámbito educativo, entre las que destacan: en 2005 la educación fue elegida por Brasil como una herramienta importante para superar la homofobia en la Convención sobre la Protección y Promoción de la Diversidad de las Expresiones Culturales celebrada en París (PEREIRA; BAHIA, 2011); en 2007, la cuestión de la diversidad afectivo-sexual se incluyó como tema transversal en el plan de estudio de las escuelas (BRASIL, 2007, p. 32-33). Esta propuesta está presente en la legislación internacional sobre derechos humanos LGBT+ (PRINCIPIOS DE YOGYAKARTA, 2007, p. 22-23); en 2008, la propuesta de estrategias para combatir la homofobia (BRASIL, 2008) y el posterior análisis y evaluación dio lugar al fortalecimiento de las directrices para la lucha contra la homofobia (BRASIL, 2011); en 2009, el incentivo para el desarrollo de políticas para la promoción, visibilidad y reconocimiento de este colectivo fue destacado en el Programa Nacional de Derechos Humanos (PNDH-3) en el objetivo estratégico V (BRASIL, 2010, p. 98-99).

En 2014, el Plan Nacional de Educación (PNE) presenta, a través de la Ley Federal no 13.005/2014 (BRASIL, 2015), las directrices, metas y estrategias para las políticas educativas en todo Brasil para un período de diez años. El PNE actual tiene validez hasta 2024 y conlleva un total de 20 objetivos. Las palabras género, orientación sexualy bullying no aparecen en el texto final. Las palabras género y orientación sexual fueron suprimidas por la presión de grupos conservadores, dirigidos por políticos evangélicos en el Congreso Nacional. Entre las directrices establecidas que figuran en la PNE actual, se observa que lo más cercano a los conceptos mencionados son los puntos III y X del artículo 2, cuando se establece que:

Art. $2^{\circ}$ São diretrizes do PNE: III - Superação das desigualdades educacionais, com ênfase na promoção da cidadania e na erradicação de todas 
as formas de discriminação; X - Promoção dos princípios do respeito aos direitos humanos, à diversidade e à sustentabilidade socioambiental. (BRASIL, 2015, p. 12-13) ${ }^{3}$.

Cabe señalar que la PNE marca la Base Nacional Curricular Común (BNCC), que es la política educativa pública que establece los conocimientos y habilidades de los planes de estudio, que las/os estudiantes brasileñas/os deben adquirir a lo largo de su educación escolar. Es decir, es un documento que guía el currículo escolar. $\mathrm{Al}$ igual que en la PNE, cabe destacar que la BNCC fue aprobada (en diciembre de 2017) con la supresión de los términos "género" y "orientación sexual", al mismo tiempo que hubo la inclusión de la educación religiosa. Los sectores conservadores de la sociedad celebraron esta decisión, ya que abogan por un currículo libre de "ideología de género", un término y un movimiento inicialmente construido por la Iglesia Católica y a posteriori difundido por religiones y grupos conservadores, especialmente por la extrema derecha, que utilizan ese término para combatir la igualdad de derechos en todos los aspectos relacionados con el género y la sexualidad (NARDI; MACHADO; SILVEIRA, 2015). Estos sectores, en su mayoría de orientación religiosa fundamentalista, consideran que el género es un atributo biológico marcado por los genes y que tratarlo en los planes educativos significaría defender ideologías y/o adoctrinamientos en las escuelas e institutos brasileños.

El BNCC fue un experimento de construcción participativa de una política de Estado. Durante este proceso de 4 años, hubo discusiones y estudios de decenas de personas expertas en el área de la educación, con conferencias regionales y un amplio debate en la sociedad civil. Sin embargo, esta política también fue atacada y desvirtuada de su idea original ya en su fase final de construcción

Art. $2^{\circ}$ Son directrices de la PNE: III - Superación de las desigualdades educativas, con énfasis en la promoción de la ciudadanía y la erradicación de todas las formas de discriminación; X - Promoción de los principios del respeto a los derechos humanos, a la diversidad y a la sostenibilidad socioambiental (BRASIL, 2015, p. 12-13). 
en 2016 (ANPED, 2018); es decir, un período en el que la frágil democracia de Brasil comenzó a ser duramente golpeada.

Después de 2016, los gobiernos, con el apoyo del Congreso Nacional, iniciaron una ofensiva contra la "ideología de género". Esto llegó incluso a la cooptación de la diplomacia brasileña que, en 2019, recibió instrucciones para, en el Consejo de Derechos Humanos de la Organización de las Naciones Unidas (ONU), reiterar y enfatizar que la palabra género significa sexo biológico: es decir, hombre y mujer, alineados con una minoría de países dirigidos por gobernantes musulmanes fundamentalistas que comparten esta posición. En consecuencia, diferentes grupos de investigación, instituciones, movimientos sociales y organizaciones de derechos humanos en todo Brasil están señalando las distorsiones que el concepto de género y la noción de "igualdad de género" están sufriendo. Estos debates tienen lugar tanto en el ámbito público, como en el educativo y en la política interna y externa del país.

Tratar el género y la sexualidad no es destruir la familia, ni querer eliminar los preceptos morales del concepto de familia. Es solo presentar una diversidad de formas de ser y de existir en nuestro mundo. Este tema, cuando se aborda en el entorno escolar, además de contribuir a la reducción del bullying homofóbico, facilita la creación de entornos de respeto y capacita a las personas para abordar la diversidad. Por lo tanto, las necesidades de la población LGBT+ deben ser trabajadas en el entorno escolar para que las situaciones de violencia puedan, a través de la construcción de una cultura inclusiva, transformarse en oportunidades de aprendizaje.

La exclusión del debate sobre el género implica el no cumplimiento de uno de los objetivos de la educación, que es el respeto a la diversidad humana. Al eliminar un tema transversal de los planes de estudio de los centros educativos se impide el reconocimiento de identidades que difieran de la cisheteronormatividad. No tener en cuenta esos conceptos en estos planes de educación refuerza el aislamiento de grupos históricamente excluidos. Además, contribuye al mantenimiento de prejuicios y discriminaciones que asignan, por 
ejemplo, a las mujeres, a la población LGBT+, a la diversidad funcional o las poblaciones negras e indígenas, un carácter de inferioridad, que impediría su acceso al sistema escolar. Según Dayana Brunetto (2019), la ideología de género es una estrategia de poder criminal que desactiva y silencia la confrontación de la violencia en las escuelas. Este panorama es preocupante, ya que la escuela es tras la familia - el principal núcleo de socialización del ser humano.

Esta acción, de alcance nacional e internacional, es una forma de violencia institucional de una estructura social que alienta a los grupos conservadores fundamentalistas a movilizarse para hacer valer sus posiciones y prohibir discusiones acerca de los conceptos de género y orientación sexual en los planes nacionales, estatales y municipales de educación. Para ejemplificar tal argumento, antes de la implementación de la PNE, se puso en marcha un proyecto financiado por el Ministerio de Educación llamado Escola sem Homofobia (BRASIL, 2011), que tenía como objetivo llevar a cabo acciones que promuevan entornos políticos y sociales favorables a garantizar los derechos humanos y el respeto a las orientaciones afectivo-sexuales y la identidad de género en el entorno escolar brasileño, a través de la distribución de un material educativo dirigido a la educación secundaria conocida como "kit educativo anti-homofobia". Este proyecto de ley fue vetado por la presión popular y la articulación política del sector evangélico del Senado Federal (NARDI, 2018). Los derechos humanos LGBT+ han estado acompañados por un intenso debate en los sectores más conservadores de la sociedad impregnados del discurso político que ha vetado estos temas en las escuelas e institutos brasileños. Esto acaba repercutiendo y fomentando la violencia contra las personas LGBT+ en el entorno escolar, incluyendo el bullying homofóbico.

\subsection{Panorama político actual y su relación con la población LGBT+}

En Brasil, los logros relacionados con la población LGBT+ provienen más del Poder Judicial que del Legislativo (NARDI; 
KVELLER; MACHADO, 2018). Esto demuestra cómo los gobernantes que son, en teoría, elegidos por el pueblo, no son capaces y no están interesados en satisfacer las demandas de los grupos sociales con menor representación. Esto dice mucho acerca del desempeño del Congreso Nacional brasileño y la democracia burguesa que no concede a todas las personas las mismas condiciones de competitividad en los procesos electorales dada la baja representación de las mujeres, los pueblos indígenas, las personas negras y LGBT+ en el Congreso Nacional. Actualmente, ciertas propuestas legislativas que podrían representar logros significativos para estos grupos sociales, a veces no se ponen en la agenda o no obtienen la cantidad de votos necesarios para su aprobación.

Con la llegada de las redes sociales y la comunicación prácticamente instantánea, las personas tienen acceso inmediato a todo tipo de información. Eso acaba potenciando no sólo el alcance, sino también la influencia sobre la ciudadanía de las declaraciones realizadas. Este proceso se intensifica cuando esos discursos son proferidos por cargos electos - como los actuales gobernantes de Brasil - actores sociales supuestamente elegidos para representar la voluntad de toda la ciudadanía.

Es a través de las noticias y las informaciones de las redes sociales que la degradación de la protección de los derechos humanos, incluidos los de la población LGBT+, se hace notoria. Se observa que la ciudadanía y los partidos políticos conservadores demuestran cada vez más la proyección egoísta y meritocrática de las situaciones individuales de privilegio, a través del desprecio de las supuestas minorías y sus luchas, en favor de una realidad que no observa y comprende el mundo que lo rodea, considerando las experiencias individualizadas de una minoría blanca de clase alta y media alta como si correspondieran a la nación.

A diario se publican innumerables noticias en los múltiples canales de comunicación, sobre las agresiones a los derechos humanos de estas minorías, incluidos las/os niñas/os que no se ajustan a las normas consideradas correctas por sus familias cishetero- 
normativas. Ante este escenario, las publicaciones muy criticadas y poco respetuosas realizadas por la extrema derecha, posibilitadas por la abdicación de lo que una vez pudo llamarse una derecha republicana, refuerzan las prácticas violentas y excluyentes, ya que encuentran un eco en las opiniones de quienes deben representar y defender los derechos humanos de toda la ciudadanía.

En 2018, en un artículo publicado por El Pais ${ }^{4}$, se compilaron declaraciones de Jair Bolsonaro en sus casi 30 años de carrera política que destacan el machismo y las expresiones de odio en sus pronunciamientos. Como ocupante del más alto cargo del Poder Ejecutivo, el actual presidente de la República de Brasil sigue pronunciando y reforzando su discurso de odio.

Sin embargo, las acusaciones cargadas de prejuicios no se limitan únicamente a la figura del actual presidente. A modo de ejemplo, la ministra de Mujeres, Familia y Derechos Humanos, Damares Alves, afirmó que Brasil estaba entrando en una "nueva era", en la que "los niños se vestirán de azul y las niñas vestirán de rosa". Estos discursos refuerzan los estereotipos de género y la cisheteronormatividad, con consecuencias en el entorno escolar, contribuyendo a los prejuicios y el rechazo y al aumento de las tasas de enfermedad mental y suicidio estructural de este colectivo social. En 2019 se contabilizaron 329 asesinatos y suicidios estructurales contra LGBT+ (OLIVEIRA; MOT'T, 2020). Frente a estas declaraciones, es evidente que la incitación al odio ha sido legitimada por quienes han sido elegidos y que constitucionalmente deberían proteger los derechos humanos y fundamentales de toda la población del país, sin excepción alguna.

Este es un aspecto fundamental, ya que las actitudes hacia las personas LGBT+ dependen básicamente de cómo están siendo discursivamente construidas en los discursos dominantes. De aquí que un elemento clave para prevenir las actitudes homófobas sea la

$4 \quad$ O que Bolsonaro já disse de fato sobre mulheres, negros e gays ( 07 de outubro de 2018). El País. https://brasil.elpais.com/brasil/2018/10/06/politica/1538859277_033603.html 
existencia de un discurso por parte de los líderes políticos y mediáticos inclusivo que enfatice en positivo la diversidad y la igualdad en derechos de todas las personas. Como señala Cachón (2011), en el tema de la inmigración, se necesitan buenas políticas (en educación, economía, vivienda, servicios sociales...) para combatir la exclusión social, pero estas buenas políticas no son suficientes por sí solas. Es necesaria también una posición clara y positiva por parte del Gobierno, de los políticos y los personajes públicos, que reconozca la dignidad y las contribuciones a la sociedad que realizan las personas LGTB+.

La elección de un presidente de la República y de miembros del Congreso Nacional con discursos cargados de prejuicios legitima a quienes comparten la misma forma de pensar y ejercer su opresión contra dichas minorías. Estas posiciones se reflejan en el funcionamiento de las instituciones. Este conjunto de situaciones demuestra cómo los derechos conquistados no son definitivos. Es un acto constante de resistencia por parte de las personas que están del lado de los derechos humanos y que buscan una sociedad donde haya justicia y oportunidades para que todos desarrollen su potencial sin verse marginados de las políticas estatales.

A pesar de las constantes declaraciones públicas que incitan al odio y la violencia y vulneran los derechos humanos LGBT+, el país ha tomado algunas medidas en la lucha contra la LGBTfobia. El Tribunal Supremo Federal (STF), después de tres meses de debate, decidió, el 13 de junio de 2019, que la discriminación por orientación afectivo-sexual e identidad de género sea un delito. La magistratura de la Corte Suprema determinó que la conducta es ahora castigada, incluso provisionalmente, por la Ley del Delito de Racismo (Ley no 7716/1989), que prevé delitos de discriminación por raza, "color", etnia, religión y origen. En Brasil, el delito de racismo no prescribe y puede ser castigado con una pena de uno a cinco años de prisión y, en algunos casos, mediante sanción administrativa (IOTTI, 2019). En resumen, la situación política actual tiene un impacto en la forma en que la población LGBT+ es tra- 
tada en las escuelas, ya que la educación en la diversidad promueve modelos masculinos y femeninos más inclusivos y saludables, así como el reconocimiento del otro y aprender a vivir en sociedad.

\section{La vulneración de los derechos LGBT+ en el ámbito educativo: el bullying homofóbico}

Estudios sobre la desigualdad de género y su relación con la educación (ABGLT, 2016; SANTOS; CERQUEIRA-SANTOS, 2020), señalan que el espacio escolar es un entorno en el que se observan cada vez más situaciones de agresividad e indisciplina. El entorno escolar es un contexto hostil para todas las personas que no performan las normas cisheterosexuales establecidas de identidad, afectividad y sexualidad. Lo anterior ha desencadenado procesos institucionalizados de prejuicio, discriminación, crímenes de odio y exclusión escolar.

El bullying homofóbico está incrustado en los institutos de todo el mundo. Este fenómeno se define como una violencia de grupo de carácter heterosexista y cisheteronormativo ejercida de forma intencional y continuada sobre una persona, al que un grupo de iguales agrede, amenaza, humilla, rechaza y/o culpabiliza en base a su orientación afectivo-sexual homosexual real o percibida (VICENTE DA CRUZ, 2019). El bullying homofóbico es una forma de violencia que implica a toda la comunidad educativa, que asumen diferentes roles que pueden mantener o frenar la dinámica de acoso en función de cuál sea su actuación.

El bullying homofóbico es un problema relevante en sus diferentes formas de expresión. El escenario académico indica que el lenguaje homofóbico, que es percibido como inofensivo por ser parte de la jerga juvenil (ABGLT, 2016; ESPELAGE et al., 2018b; SANTOS; CERQUEIRA-SANTOS, 2020), es lo que facilita el desarrollo de mecanismos estigmatizadores y deshumanizadores que conspiran contra sus víctimas (ESPELAGE et al., 2018b; RIVERS, 2011; RONDINI; TEIXEIRA-FILHO; TOLEDO, 2017); es decir, la ironía, el sarcasmo, los chistes, las burlas, las bromas, representa- 
ciones caricaturescas y burlescas, etc. El uso del lenguaje homofóbico para atacar, oprimir, menospreciar, humillar e infra humanizar a las víctimas es un fenómeno habitual en los centros escolares. La naturalización del lenguaje homofóbico en los institutos de Brasil revela el actual orden moral y ético de esa sociedad.

El poder del lenguaje homofóbico es tan devastador que los oprimidos reconocen y aceptan los instrumentos que les oprimen y terminan asumiendo el estatus social de desviado y construyen su identidad mediante la internalización del estigma sexual (HEREK, 2015). Por esta y otras razones, la resignación, el aislamiento social, la omisión, las conductas autolesivas y autodestructivas, la promoción de la cisheteronormatividad, la socialización con los cómplices del acoso, la invisibilización de la propia homosexualidad, la utilización de la burla homofóbica, la reproducción del bullying con otras víctimas, la agresión defensiva etc. son algunas de las estrategias usadas para remediar el acoso (VICENTE DA CRUZ, 2019, 2021). En general, las personas afectadas por la violencia escolar suelen elegir estrategias desadaptativas para afrontar el acoso padecido (WÓJCIK et al., 2021).

El lenguaje homofóbico no se dirige sólo a lesbianas y a los gais (RODRÍGUEZ-HIDALGO; HURTADO-MELLADO, 2019). En este contexto, es empleado en especial cuando existe la sospecha gay. Como el lenguaje homofóbico también es guiado por determinadas actitudes, conductas y apariencias, los chicos percibidos como débiles, pacíficos o inteligentes, que no les gustan los deportes colectivos o que transgreden los códigos de vestimenta específicas del contexto sufrirán bullying homofóbico (PLUMMER, 2001). El humor anti-gay es una estrategia empleada como respuesta a una amenaza de la masculinidad tradicional (O'CONNOR; FORD; BANOS, 2017). Por esto, los chicos suelen ejercer y sufrir más acoso homofóbico que las chicas (APA, 2018; RIVERS, 2011). Esos resultados confirman la fuerza y el poder de la socialización del género masculino.

El bullying homofóbico, así como otras formas de violencia que sufren LGBT+ en el contexto educativo, posee tres caracterís- 
ticas específicas respecto a otros tipos de acoso escolar: el rechazo de la familia y la falta de apoyo social; su invisibilización en el sistema educativo; y la naturalización y normalización de la homofobia en la sociedad.

El ámbito familiar suele ser el lugar donde la persona LGBT+ vive por primera vez la homofobia. La familia puede castigarla a través de la homofobia sutil (bromear, excluir, etc.) o manifiesta (agredir, insultar, etc.). Por esto, pedir apoyo a un familiar que no sea LGBT+ puede ser complicado y problemático para las personas afectadas por este fenómeno, ya que muchas veces no se sabe cómo reaccionará (PLUMMER, 2001). La homofobia en la familia es deshumanizadora, quedando invisibilizada en uno de los entornos más íntimos de la víctima.

Según Borrillo (2001), a diferencia de otras formas de violencia, el individuo que experimenta homofobia puede sufrir en soledad el ostracismo, sin ningún tipo de apoyo de su entorno, incluyendo el familiar. Por esto, estas personas son fácilmente víctimas del desprecio y comúnmente internalizan el estigma sexual (HEREK, 2015).

El sistema educativo invisibiliza el bullying homofóbico con el silencio. El silencio es una forma de maltrato que encubre, autoriza y legitima la violencia. Uno de los elementos que explicaría la pasividad de las autoridades educativas es precisamente la normalización de la homofobia en las sociedades contemporáneas. El bullying homofóbico podría ser combatido e, incluso, erradicado, si hubiera una intervención eficaz de las instituciones educativas.

La normalización del bullying homofóbico solo es posible debido a la impunidad, minimización, deshumanización y el silencio de las autoridades políticas, religiosas, comunitarias y educativas (ANTÓNIO; GUERRA; MOLEIRO, 2017; ESPELAGE et al., 2018a; RIVERS, 2011; RODRÍGUEZ-HIDALGO; HURTADO-MELLADO, 2019). La LGBTfobia es una de las discriminaciones que están inscritas en el orden jurídico de diversos países (ILGA, 2019). Incluso en países que no tienen leyes que criminalizan a la 
población LGBT+, como Brasil, las autoridades políticas, como la actual presidencia de la República, propagan públicamente el odio contra ese colectivo.

Una revisión sistemática de los estudios de los últimos 20 años sobre homofobia y escuela en Brasil (SANTOS; CERQUEIRA-SANTOS, 2020), señala el entorno escolar como un ambiente propicio para este fenómeno. Los estudios citados en esta revisión indican los siguientes resultados similares: el alumnado desea mantener la distancia de las personas LGBT+; el profesorado no tiene competencias para abordar la educación sexual en el aula; la naturalización del lenguaje homofóbico. Los datos del último estudio nacional realizado en Brasil sobre "experiencias de adolescentes y jóvenes LGBT+ en el contexto escolar" entre 2015 y 2016 con 1.016 jóvenes LGBT+ de 13 a 21 años, indican que: el $60 \%$ de la juventud estudiada se siente insegura en la escuela; el $48 \%$ escuchó comentarios homofóbicos del grupo de iguales; el $73 \%$ fue agredida verbalmente; y el 27\% fue agredida físicamente (ABGLT, 2016).

El bullying homofóbico puede tener numerosas consecuencias negativas para la salud de sus víctimas. Entre las consecuencias más drásticas se encuentran el suicidio estructural, el abuso de sustancias, las conductas sexuales de riesgo, vulnerabilidad a enfermedades de transmisión sexual como el VIH y la sífilis, depresión, ansiedad, baja autoestima y estrés postraumático. También puede tener implicaciones psicosociales importantes, como el aislamiento social, el absentismo y el abandono escolar prematuro, lo que conllevará menos oportunidades de integración, riesgo de exclusión social y pone en entredicho el bienestar.

El acoso escolar homofóbico puede ser reforzado por la falta de políticas públicas educativas, ya que esta ausencia implica la prohibición de las discusiones sobre los derechos humanos LGBT+ y la construcción de nuevas subjetividades en las escuelas. El debate sobre el género como tema transversal en las escuelas e institutos brasileños permitiría conocer los diversos mecanismos 
para detectar y/o evitar el bullying homofóbico, además de revelar por qué este tipo de violencia podría normalizarse y legitimarse.

Este problema refuerza la importancia de una educación basada en una cultura crítica, contra modelos que reproducen las represiones y a favor de una educación que asuma la existencia de la diversidad humana, donde diversas prácticas y deseos afectivos-sexuales sean posibles e igualmente válidos. No se trata de educar a las/os niñas/os y jóvenes para que se expresan fuera de la cisheteronormatividad ni para que se normalicen en la sociedad, sino de enseñarles a entender la diversidad como parte de la humanidad para que se pueda construir una cultura inclusiva que llegue a todas y a todos con sus singularidades. Por esta razón, es esencial reconocer la importancia de la (re) inclusión de los conceptos de género y diversidad sexual y la inclusión del concepto de bullying (o la versión portuguesa del concepto - "assédio”) en el PNE y BNCC, ya que esto implicaría tratar el respeto por los demás y promover la formación a través de políticas que tienen en cuenta la noción de igualdad de género. La normalización de las diversidades como parte de la condición humana empieza en la educación, porque sólo con la naturalización de las diversidades es posible actuar contra la opresión. Promover la construcción de ambientes seguros y amables, acogedores y educativos de manera efectiva, para apuntar a políticas orientadas hacia el respeto, la aceptación y la comprensión de la diversidad, proporcionando mayores habilidades transversales al profesorado y al alumnado, y asegurando una educación carente de opresiones son prioridades, ya que la diversidad es un recurso liberador, dotado de un gran potencial pedagógico.

En resumen, reivindicar la igualdad afectivo-sexual y de género en el sistema educativo significa crear acciones específicas para combatir todo tipo de violencia que contribuya a la reproducción de las desigualdades que persisten en Brasil. Esta garantía es esencial, ya que la experiencia escolar puede y debe desempeñar un papel central en los conceptos que promueven la igualdad, la equidad y la diversidad humana, así como la deconstrucción y transformación 
de mitos e ideas preestablecidas que constituyen un referente normativo en la que se articulan los discursos y prácticas que sostienen las dominaciones y las relaciones de poder. El derecho a la educación y a los procesos educativos comprometidos con la equidad debe garantizarse a cualquier persona, independientemente de su origen étnico-racial, diversidad afectivo-sexual y de género, diversidad funcional, estatus socioeconómico, creencias religiosas, etc.

\section{Conclusiones}

El bullying homofóbico es producto de múltiples factores, como el rechazo de la familia, su invisibilización en el sistema educativo y su naturalización en la sociedad. Por esto, es necesario realizar intervenciones psicosociales en estos ámbitos para potenciar los factores de protección a nivel individual, grupal, familiar, educativo y social (ANTÓNIO; GUERRA; MOLEIRO, 2017; ESPELAGE et al., 2018a; NAPPA et al., 2017; PACE; D’URSO; ZAPPULLA, 2021; RODRÍGUEZ-HIDALGO; HURTADO-MELLADO, 2019).

Las familias con niñas/os y jóvenes LGBT+ deben adquirir habilidades y competencias educativas, comunicativas y sociales y saber cuestionar su visión sobre lo que es la diversidad (PACE; D’URSO; ZAPPULLA, 2021). Además, tienen que tomar conciencia de su influencia en el desarrollo de la persona LGBT+ para acentuar su función protectora. La familia debe ser una aliada de la diversidad y aceptarlo incondicionalmente (ESPELAGE et al., 2018a). Para ello, los familiares deben adquirir herramientas pedagógicas y educarse en la diversidad.

Los centros educativos deben romper con el silencio y fomentar políticas orientadas al respeto y la aceptación de la diversidad (NAPPA et al., 2017). Eso empoderará al alumnado y le proporcionará competencias transversales para que rompa con su silencio, defienda las víctimas y detecte precozmente el bullying homofóbico. Hay estudios que indican que quien defiende a las víctimas es percibido de forma positiva por los iguales, es considerado empático, emocionalmente estable y cognitivamente competente (ANTÓ- 
NIO; GUERRA; MOLEIRO, 2017; ESPELAGE et al., 2018b; RODRÍGUEZ-HIDALGO; HURTADO-MELLADO, 2019).

Sobre las acciones de resistencia a estas medidas de involución dentro de los centros educativos, la eliminación de los términos (tal como se ha hecho en la PNE) no impide que las/os docentes trabajen estos problemas en el aula. En estos casos, son los actos del profesorado o de los centros educativos que estarían atentos a su alumnado y a sus problemas. Es decir, las/os niñas/os y jóvenes LGBT+ podrían encontrar la acogida en la escuela que a menudo no encuentran en la familia. De lo contrario, estarán condenados a la violencia que la cisheteronormatividad impone en el entorno escolar.

El bullying homofóbico es un fenómeno dinámico que se transforma y se amolda a las idiosincrasias de cada contexto. Por eso, si los agentes políticos y sociales mantuvieran relaciones estrechas con las personas LGBT+ y promovieran su aceptación y el trato igualitario, los prejuicios sociales tan enraizados en Brasil se reducirían. Por esto, declaraciones que incitan el odio a grupos históricamente estigmatizados son tan nocivas. Este ensayo crítico advierte a esos agentes que tengan presente que sus declaraciones prejuiciosas incitan el odio contra las minorías sociales, incluyendo la población LGBT+.

Este ensayo concluye con la esperanza de que es posible vivir en una sociedad donde el trato igualitario hacia la diversidad humana es parte de las praxis sociales cotidianas. Por lo tanto, es necesario identificar el alcance de todos los tipos de violencias en el contexto escolar, realizar políticas de sensibilización, involucrar a la comunidad escolar y desarrollar distintas formas de intervención en el bullying homofóbico para su prevención y detección precoces.

\section{Referencias}

AMERICAN PSYCHOLOGICAL ASSOCIATION (APA). Boys and men guidelines group. APA guidelines for psychological practice with boys and men. 2018.

ANTÓNIO, R.; GUERRA, R.; MOLEIRO, C. Having friends with gay friends? The role of extended contact, empathy and threat on assertive bystanders' behav- 
ioral intentions. Revista de Psicologia, 31(2), p. 15-24. 2017. DOI https://doi. org/10.17575/rpsicol.v31i2.1138.

ASOCIACIÓN INTERNACIONAL DE LESBIANAS, GAIS, BISEXUALES, TRANS E INTERSEX (ILGA). Homofobia de Estado 2019. Ginebra: ILGA, 2019.

ASSOCIAÇÃO BRASILEIRA DE LÉSBICAS, GAYS, BISSEXUAIS, TRAVESTIS E TRANSEXUAIS (ABGLT). Pesquisa nacional sobre o ambiente educacional no Brasil 2015: as experiências de adolescentes e jovens lésbicas, gays, bissexuais, travestis e transexuais em nossos ambientes educacionais. Curitiba, Brasil: Secretaria de Educação, 2016.

ASSOCIAÇÃO NACIONAL DE PÓS-GRADUAÇÃO E PESQUISA EM EDUCAÇÃO (ANPED). A proposta de BNCC do ensino médio: alguns pontos para o debate. 2018.

BORRILLO, D. Homofobia. Barcelona, España: Bellaterra, 2001.

BRASIL. $2^{\text {a }}$ Conferência Nacional de Políticas Públicas e Direitos Humanos de LGBTI. Brasília, Brasil: Secretaria Especial dos Direitos Humanos da Presidência da República, 2011.

BRASIL. Comitê Nacional de Educação em Direitos Humanos. Plano nacional de educação em direitos humanos. Brasília, Brasil: Secretaria dos Direitos Humanos, MEC, MJ, 2007.

BRASIL. Direitos Humanos e Políticas Públicas: o caminho para garantir a cidadania de Gays, Lésbicas, Bissexuais, Travestis e Transexuais. Anais da Conferência Nacional de Gays, Lésbicas, Bissexuais, Travestis e Transexuais. Brasília: Secretaria Especial dos Direitos Humanos da Presidência da República, 2008.

BRASIL. Ministério da Saúde. Conselho Nacional de Combate à Discriminação (SEDH). Brasil sem homofobia: programa de combate à violência e à discriminação contra GLTB e de promoção da cidadania homossexual. Brasil, 2004.

BRASIL. Plano Nacional de Educação PNE 2014-2024: linha base. Brasília: Ministério de Educação (MEC), Instituto Nacional de Estudos e Pesquisas Educacionais Anísio Teixeira (Inep), 2015.

BRASIL. Programa Nacional de Direitos Humanos (PNDH-3). Brasília, Brasil: Secretaria de Direitos Humanos da Presidência da República, 2010. 
BRUNETTO, D. Por uma educação que respeite as interseccionalidade e enfrente o bullying e a violência contra LGBTI+. En T. Reis Congresso Internacional LGBTI+. Curitiba, Brasil, nov. 2019.

BULGARELLI, L. et al. Violência contra LGBTs+ nos contextos eleitoral e pós-eleitoral. Brasil: Gênero e Número, 2019.

CACHÓN, L. (2011). Inmigración y conflictos en Europa. Aprender para una mejor convivencia. Barcelona, España: Editorial Hacer.

ESPELAGE, D. et al. A literature review of protective factors associated with homophobic bullying and its consequences among children \& adolescents. Aggression and Violent Behavior, 45, p. 98-110. 2018a. DOI https://doi. org/10.1016/j.avb.2018.07.003.

ESPELAGE, D. et al. Longitudinal examination of the bullying-sexual violence pathway across early to late adolescence: implicating homophobic name-calling. Journal Youth Adolescence, 47, p. 1880-1893, 2018b. DOI https://doi. org/10.1007/s10964-018-0827-4.

HEREK, G. Beyond "homophobia": Thinking more clearly about stigma, prejudice, and sexual orientation. American Journal of Orthopsychiatry, 85(5), p. 29-37. 2015. DOI https://doi.org/10.1037/ort0000092.

IOTTI, P. Advocacia no Judiciário. As conquistas LGBTI+ no STF. Reis Congresso Internacional LGBTI+. Curitiba, Brasil, nov. 2019.

MCCONNELL, E. et al. "Everybody puts their whole life on Facebook": identity management and the online social networks of LGBTQ youth. International journal of environmental research and public health, 15(6), p. 1078-1096, 2018. DOI https://doi.org/10.3390/ijerph15061078.

NAPPA, M. et al. Teachers' Reaction in homophobic bullying incidents: the role of self-efficacy and homophobic attitudes. Sex Res Soc Policy, 15, p. 208-218, 2017. DOI https://doi.org/10.1007/s13178-017-0306-9.

NARDI, H. C. Politiques publiques et pluralités sexuelles et de genre au Brésil. Le contexte général et les spécificités de l'éducation. In: PERRIER, L. (org.). L’altérité et l'identité à l'épreuve de la fluidité. 1. ed. v. 1, p. 159-182. Paris: L'Harmattan, 2018.

NARDI, H. C.; GONCALVES, R. A. S. Infância, religião e colonialidade: trajetórias de homens gays. Momento - Diálogos em Educação, v. 28, p. 195-207, 2019. DOI https://doi.org/10.14295/momento.v28i1.8765. 
NARDI, H. C.; KEVLLER, D.; MACHADO, P. S. Direitos Humanos e os marcadores sociais da diferença: desafios do cenário brasileiro. In: NARDI, H. C.; ROSA, M. V. F.; MACHADO, P. S.; SILVEIRA, R. da S. (org.). Políticas públicas, relações de gênero, diversidade sexual e raça na perspectiva interseccional. 1. ed. v. 1. Porto Alegre: Secco, 2018.

NARDI, H. C.; MACHADO, P. S.; SILVEIRA, R. da S. (org.). Diversidade sexual e relações de gênero nas políticas públicas: o que a laicidade tem a ver com isso. 1. ed. v. 1. Porto Alegre: ABRAPSO/DERIVA, 2015.

NARDI, H. C.; QUARTIERO, E.; RODRIGUES, M. C. Queer intersectional outreach actions to prevent $\mathrm{LGBTQ}^{+}$prejudice and discrimination in schools: the brazilian context and analysis of a local experience. Queer Social Movements and Outreach Work in Schools. Palgrave Macmillan, Cham, 2020. p. 157-187.

O'CONNOR, E. C.; FORD, T. E.; BANOS, N. C. Restoring threatened masculinity: the appeal of sexist and anti-gay humor. Sex Roles 77, p. 567-580, 2017. DOI https://doi.org/10.1007/s11199-017-0761-z.

OLIVEIRA, E.; MOTT, L. Mortes violentas de LGBT+ no Brasil - 2019. Relatório do Grupo Gay da Bahia, 2020.

PACE, U.; D’URSO, G.; ZAPPULLA, C. Psychological predictors of homophobic bullying among adolescents and young adults: the role of parental psychological control and sensation seeking. J Child Fam Stud. 2021. DOI https://doi. org/10.1007/s10826-020-01874-3.

PEREIRA, G.; BAHIA, A. Direito fundamental à educação, diversidade e homofobia na escola: desafios à construção de um ambiente de aprendizado livre, plural e democrático. Educar em Revista, 39, p. 51-71. 2011. DOI https://doi. org/10.1006/jado.2000.0370.

PLUMMER, D. The quest for modern manhood: Masculine stereotypes, peer culture and the social significance of homophobia. Journal of adolescence, 24(1), p. 15-23, 2001. DOI https://doi.org/10.1006/jado.2000.0370.

PRINCÍPIOS DE YOGYAKARTA. Princípios sobre a aplicação da legislação internacional de direitos humanos em relação à orientação sexual e identidade de gênero, 2007.

RIVERS, I. Homophobic bullying: research and theoretical perspective. New York, USA: Oxford University Press, 2011. 
RODRÍGUEZ-HIDALGO, A. J.; HURTADO-MELLADO, A. Prevalence and psychosocial predictors of homophobic victimizations among adolescents. Journal of Environmental Research and Public Health, 16, p. 1246. 2019. DOI https://doi.org/10.3390/ijerph16071243.

RONDINI, C. A.; TEIXEIRA-FILHO, F. S.; TOLEDO, L. G. Homophobic conceptions of high school students. Psicologia USP, 28(1), p. 57-71. 2017. DOI https://doi.org/10.1590/0103-656420140011.

SANTOS, J. J.; CERQUEIRA-SANTOS, E. Homofobia e escola: uma revisão sistematizada da literatura. Revista Subjetividades, 20 (Especial 1). 2020. DOI http://doi.org/10.5020/23590777.rs.v20iesp1.e8734.

VICENTE DA CRUZ, E. et al. Acercamiento práctico de la intervención e investigación psicosocial a la perspectiva de género. In: STREY, M. N.; CÚNICO, S. D. (org.). Teorias Feministas e Transgressão. Porto Alegre: EDIPUCRS. ISBN: 8539708550, 2016.

VICENTE DA CRUZ, E. Representaciones sociales del bullying homofóbico en adolescentes de Brasil (tesis doctoral). Universitat de Barcelona, Barcelona, España, 2019.

VICENTE DA CRUZ, E. Ser, o parecer gay, ¿cuál es la cuestión? In: GUASCH, Ò. (org.). Homofobia Académica. Barcelona, España: Edicions Bellaterra, 2021 (no prelo).

WÓJCIK, M. et al. Downward spiral of bullying: victimization timeline from former victims' perspective. Journal of Interpersonal Violence, 1-24. 2021. DOI https://doi.org/10.1177/0886260521990835.

Submetido em: 3-7-2021

Aceito em: 27-7-2021 